\title{
Subjective Image-Quality Estimation Based on Psychophysical Experimentation
}

\author{
Gi-Yeong Gim ${ }^{1}$, Hyunchul Kim ${ }^{1}$, Jin-Aeon Lee ${ }^{2}$, and Whoi-Yul Kim ${ }^{1}$ \\ ${ }^{1}$ Department of Electronics and Computer Engineering, Hanyang University, \\ Haengdang-Dong, Sungdong-Gu, Seoul, 133-792, Korea \\ \{gygim, hckim\}@vision.hanyang.ac.kr, wykim@hanyang.ac.kr \\ ${ }^{2}$ Samsung Electronics, \\ Giheung-Eup, Yongin-Si, Gyeonggi-Do, 449-712, Korea \\ jaleedsamsung.com
}

\begin{abstract}
The purpose of estimating subjective image quality is to provide best-quality image content to users in diverse fields. Image quality is subjective, therefore very difficult to estimate accurately, and so many researchers have proposed psychophysical experimentation as a means of estimating it. Conventional methods describe the relationship between the subjective preference and the perceived contrast as an "inverted U" shape. However, the relationship was resulted from only a few, high-quality images. Thus, they are inadequate for general image-quality estimation. In this paper, we carried out two experiments using a dataset with untransformed and various images. We discovered an important property that the preference increases in proportion to the perceived contrast. The result shows us not only that our experimentation can reduce MSE of image-quality estimation by approximately $40 \%$ over the previous methods, but also that it can be applied in various applications.
\end{abstract}

Keywords: Image quality, Psychophysical experimentation, Subjective quality model.

\section{Introduction}

Image quality determines the observer's level of psychophysical satisfaction. Measuring and evaluating image quality make it possible to verify the performance of image processing methods and thereby ensures that only image content of very high quality is provided for multimedia applications. The demand for accurate imagequality estimation has been increasing. However, to objectively quantize the quality of an image is a challenging task, because it is difficult to find the quality attribute that is most highly related to the perception of image quality. Thus, many researchers have proposed psychophysical-experimentation-based subjective image-quality estimation as a mean of estimating image quality [1-6].

Conventional methods [1-3] conduct experimentation using datasets generated from transformations of the following commonly employed image attributes: lightness, chroma, sharpness, noise distribution, resolution and compression. The perception of image quality is modeled with such transformed images, and thus the 
experimental results can be helpful in assessing the performance of imageenhancement algorithms. However, those experimental results are inadequate for image-quality estimation in general applications, because the image-quality models are derived from only a few, high-quality images.

As recounted in this paper, we carried out two types of experiments. The first experimentation was performed to verify the conventional methods, using a dataset generated from the transformations of the various image attributes. The second one was based on our proposed method in order to overcome the drawback of the conventional methods. To estimate subjective image quality accurately, we used datasets of various kinds of untransformed images. All experiments were conducted by the pair-comparison method, and observers were asked to choose the better image according to subjective contrast and preference. Then, to estimate the subjective quality, the perceived contrast was modeled using the image attributes, because it is difficult to approximate the subjective image quality directly from quality attributes of an image. Even if many quality attributes are used in modeling, only chroma and sharpness correlate with contrast. Hence the perceived contrast is estimated using the regression analysis with the standard deviations of chroma and sharpness. Using this model, subjective image quality is estimated according to the relationship between the perceived contrast and the preference. As a result, we found, contrary to previous experimental results, that most people prefer image of higher contrast. Moreover, compared with the conventional methods, the proposed model could reduce the mean square error (MSE) by nearly $40 \%$. This means that our model can be a significant image-content model in diverse fields.

The rest of this paper is organized as follows. In Section 2.1, we review the conventional experiment methods of estimating image quality. In Section 2.2, we describe the proposed model in detail. In Section 3, we discuss the experimental results. Finally, in Section 4 we conclude the paper.

\section{Image-Quality Experimentation}

\subsection{Related Experimentation}

Calabria and Fairchild conducted experiments to yield information relating to the perception of contrast and to develop a metric of perceived image contrast as it relates to observer preferences [1][2]. Their dataset consisted of images that were transformed for lightness, chroma, and sharpness. Because the contrast is an important attribute determining image quality, perceived contrast was modeled for image-attribute perception. To model the perceived contrast, they employed standard deviations of lightness, chroma, and sharpness. Their experimental results showed that perceived image contrast has a nonmonotonic, "inverted U"-shaped relationship to the preference. Using this relation, the preference was measured as subjective image quality. The significance of this research is that subjective quality can be estimated from the attributes of an input image. 
The Electronics and Telecommunications Research Institute (ETRI)'s CG research team in Korea proposed a method that estimates human perception based on an experimental approach [3]. Their dataset was generated by certain transformations of input images for the attributes of lightness, chroma, contrast, noise, sharpness, and compression. They conducted two types of psychophysical experiments: 'Categorical judgment' and 'Pair comparison'. The first was carried out to estimate the preference, and the second was performed to the sensitivity of human perception to image-quality attributes. The Z-scores of the results were calculated to compare the quality attributes. The Z-scores increased as a function of the transformation level until they reached the maximum, and then decreased. It means that people prefer images with middle-level lightness and chroma. They also showed that most people are more sensitive to lightness variation than chroma variation.

These researches could be valuable to performance evaluation and the setting of image processing algorithm parameters. However, their qualitative models cannot guarantee the estimated subjective image-quality result for general applications, because the dataset consists of transformed images from only a few images, in fact fewer images than are used in modeling. Therefore, in the case of evaluating images that are not used for modeling, the conventional experiments may yield inaccurate result.

\subsection{Proposed Experimentation}

The aim with this paper is to provide an experimental scheme by which subjective image quality can be estimated with high certainty. We carried out two types of experiments: Experiment I (Exp I) and Experiment II (Exp II). Exp I was performed to verify the effectiveness of the conventional methods, the dataset consisted of transformed images about image attributes. Unlike the experiments of the conventional methods [1-3], we made up a dataset that consisted of the transformed images from images of various qualities, in order to make a more general dataset than that for images only of high quality. The dataset for Exp II, the proposed method,

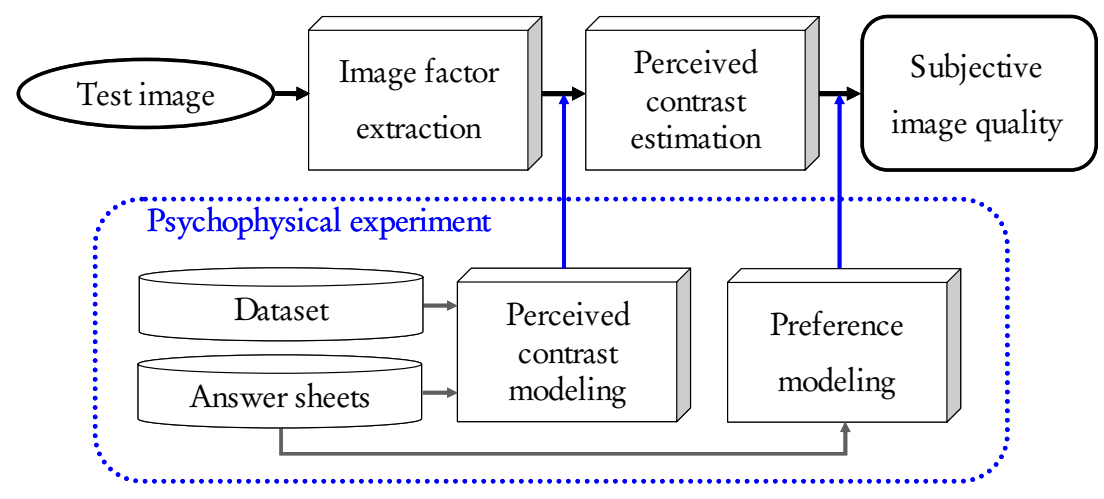

Fig. 1. Flowchart of subjective image-quality estimation 
therefore, consisted of untransformed images of various qualities. The rationale behind Exp II is that when humans estimate the quality of a certain image, they generally compare the image with different images of various qualities, the image attributes of which are not transformed.

Figure 1 shows the entire procedure for estimating the subjective image quality. As can be seen, perceived contrast modeling is performed to estimate the subjective image quality [1][2].

In Exp I, to evaluate the pair-comparison result, a total of 660 observations are generated. ${ }^{1}$ The 12 total transformations for lightness, chroma, and sharpness are listed in Table 1. In order to understand how each image attribute is independently related to the image quality, no image-quality transformation is allowed to affect other image attributes. To that purpose, transformations of the lightness and sharpness are performed on the luminance channel, and the chroma transformation is performed on the CIELAB $\mathrm{C}^{*}{ }_{\mathrm{ab}}$ channel. Figure 2 shows examples of the transformed images.

Table 1. Image-quality transformations

\begin{tabular}{|c|l|}
\hline Attributes & \multicolumn{1}{|c|}{ Transformations } \\
\hline $\begin{array}{c}\text { Lightness } \\
\text { (5 images })\end{array}$ & $\begin{array}{l}\text { Two sigmoidal functions of different shape } \\
\text { An inverse sigmoidal function } \\
\text { Two linear functions of different slope }\end{array}$ \\
\hline $\begin{array}{c}\text { Chroma } \\
(3 \text { images })\end{array}$ & $\begin{array}{l}\text { Three scaling functions with } 20 \%, 60 \%, \text { and } 120 \% \text { of the original } \\
\text { chroma }\end{array}$ \\
\hline $\begin{array}{l}\text { Sharpness } \\
(4 \text { images })\end{array}$ & $\begin{array}{l}\text { Four unsharp mask filters in Adobe Photoshop } \\
\text { and amounts of } 25,75,150, \text { and } 250\end{array}$ \\
\hline
\end{tabular}

The dataset of Exp II consists of images of various qualities, and in order to select images accurately from the worst to the best quality, we paid attention to select dataset images. Three hundred observations were made using 25 different digitalcamera images that were collected from the DPChallenge ${ }^{\circledR}$ (http://www.dpchallenge. com) and the Corbis ${ }^{\circledR}$ (http://pro.corbis.com). They contain a diverse set of high and low quality photos from many different photographers. Moreover, the images have been rated by the website users. In order to achieve highly reliable results, we performed Exp II twice with different datasets. Therefore, a total of 600 observations were used in modeling. The sample images used in Exp II are shown in Fig. 3.

Overall, 40 observers with normal color vision, consisting mainly of our faculty along with graduate and undergraduate students, participated in the experiments.

${ }^{1} 10$ images $\times C_{2}^{12}=660$, where $C_{k}^{n}=\left(\begin{array}{l}n \\ k\end{array}\right)=\frac{n !}{k !(n-k) !}$. 


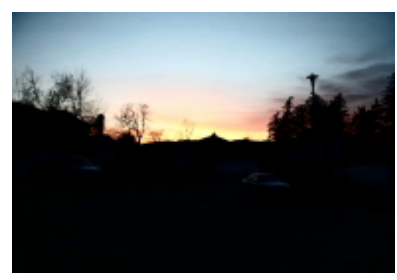

(a)

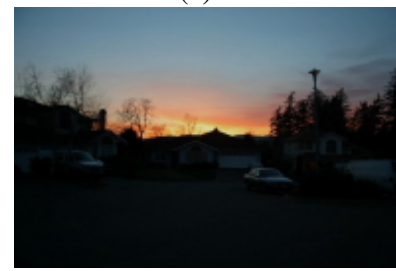

(d)

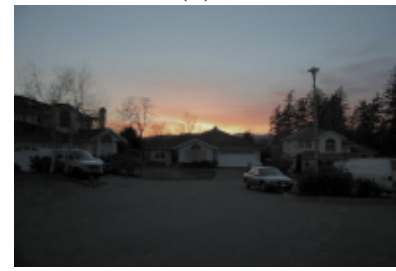

(g)

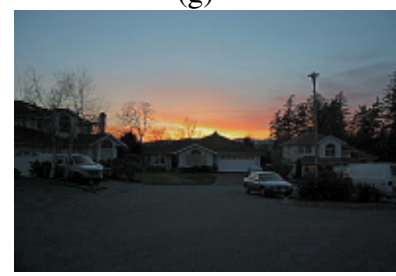

(j)

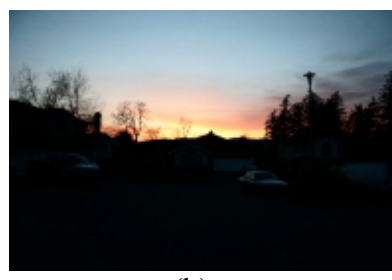

(b)

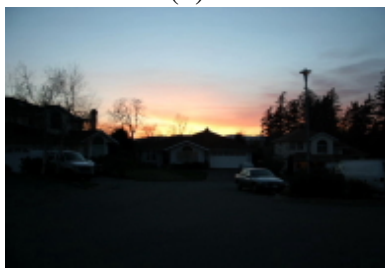

(e)

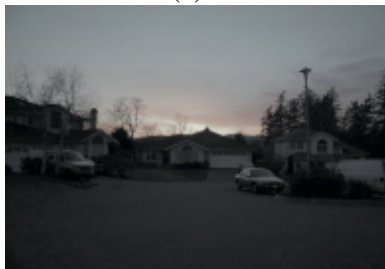

(h)

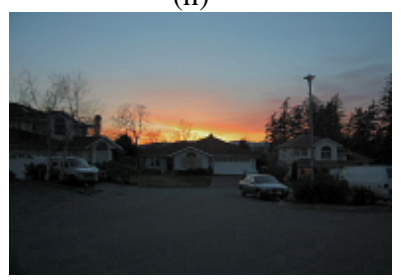

(k)

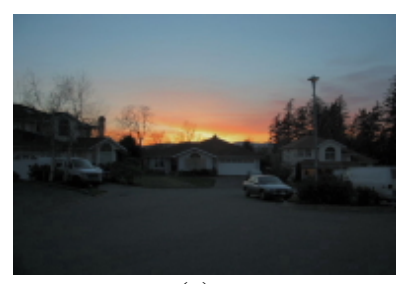

(c)

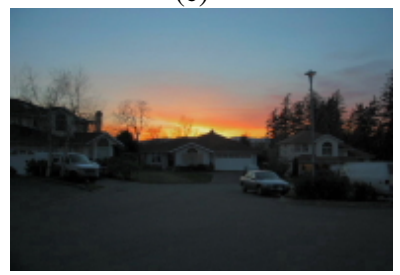

(f)

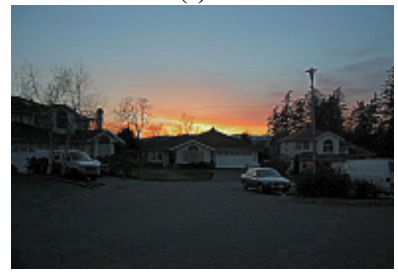

(i)

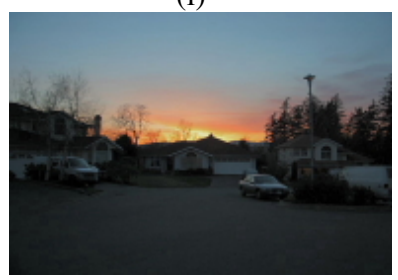

(1)

Fig. 2. Transformation examples (a)-(e) Result images for lightness: (a) Sigmoidal function with an exponent of 10, (b) Sigmoidal function with an exponent of 25, (c) Inverse sigmoidal function with an exponent of 20, (d) Linear function with a slope of 0.85 , and (e) Linear function with a slope of 1.15 (f)-(h) Result images for chroma (i)-(l) Result images for sharpness

Observer information is shown in Table 2. The observers were seated one meter from the monitor screen, and they were given no information about the purpose of the experiment. They were directed to select the better image between the two displayed images for both contrast and preference. The two-image combinations were shown on the center of a calibrated LCD monitor (Samsung SyncMaster 178B 17') in random order. The luminance of a monitor is $300 \mathrm{~cd} / \mathrm{m}^{2}$, and screen brightness level was set to $50 \%$ of the maximum [4]. The dot-pitch of monitor is $0.264 \mathrm{~mm}$ and the response time is $4 \mathrm{~ms}$. All of the experiments were performed in the same room under fluorescent lighting, and had a comfortable break (e.g. 5-10 minutes). 

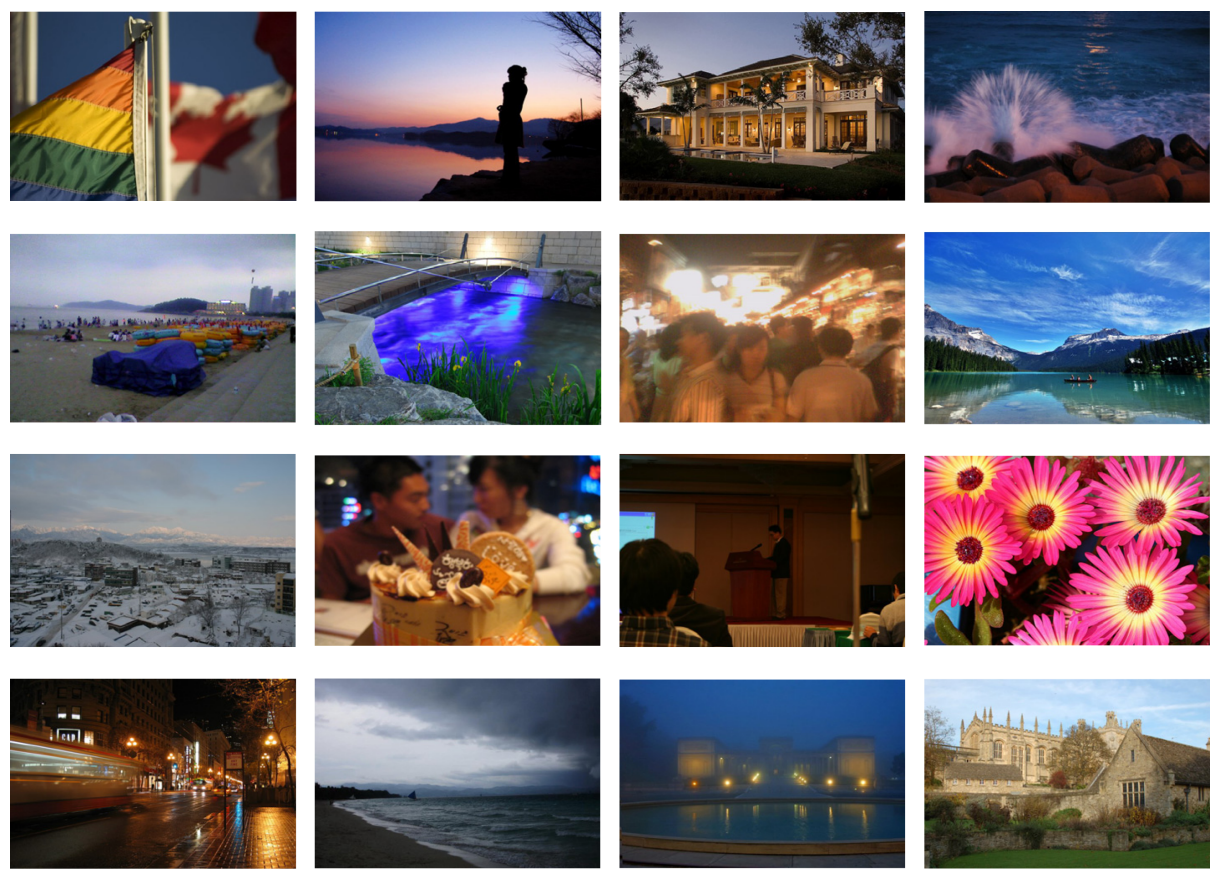

Fig. 3. The sample images using Exp II's dataset

Table 2. Observer information

\begin{tabular}{|c|c|c|c|c|c|c|}
\hline \multirow{2}{*}{$\begin{array}{c}\text { Number of } \\
\text { observers }\end{array}$} & \multirow{2}{*}{ Male } & \multirow{2}{*}{ Female } & \multicolumn{3}{|c|}{ Ethnic background } & \multirow{2}{*}{$\begin{array}{c}\text { Age } \\
\text { range }\end{array}$} \\
\cline { 4 - 6 } & & & Korean & Pakistani & Malaysian & \\
\hline 40 & 27 & 13 & 35 & 4 & 1 & $21-35$ \\
\hline
\end{tabular}

\section{Experimental Results}

To analyze the experimental results, both the preference scores and the perceived contrast scores were scaled within five levels. First of all, we analyzed the relation between the perceived preference and the subjective quality. In Fig. 4, we plot the graph of the perceived contrast versus the preference, as based on the answer sheets. Figure 4 (a) and (b) show results of Exp II using different datasets. In order to evaluate the similarity between the results, a correlation of the results was calculated. The correlation was 0.94, and it means that Exp II has a high consistency between the results. Hence we adopted averaged result for Exp II as shown in Fig. 4(d). The result of Exp I in Fig. 4(c) showed that the relationship between the perceived contrast and the preference is same as that determined by the existing methods [1-3]. The "inverted U" shape represented the fact that the highest score for the preference was around 
level 3-4. As shown in Fig. 4(d), the result of Exp II indicated that the preference is proportional to the perceived contrast. The perceived contrast of excessively transformed image brings out the decreasing preference, but untransformed image with good contrast is regarded as high-quality image.

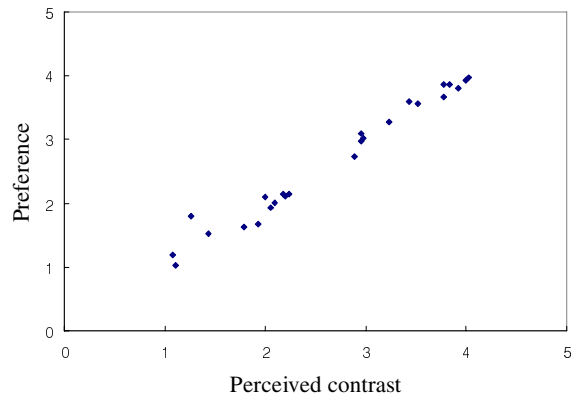

(a)

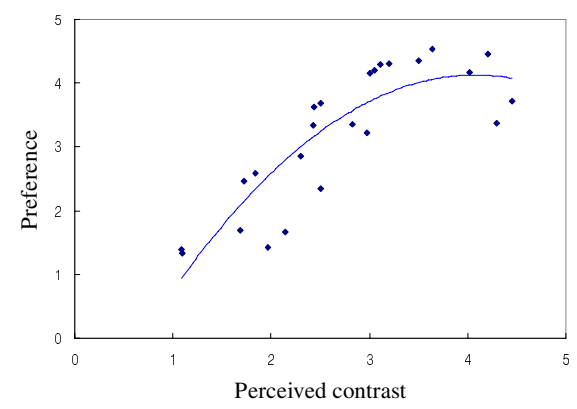

(c)

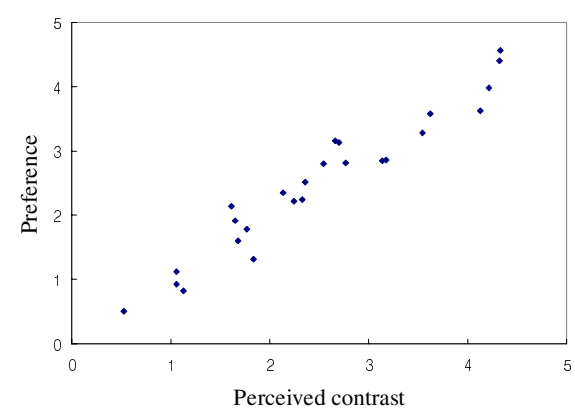

(b)

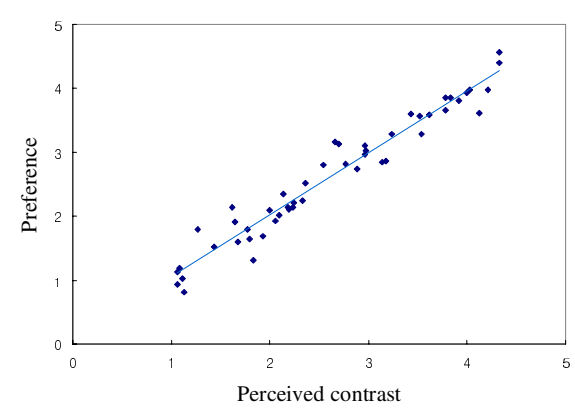

(d)

Fig. 4. The relationship between perceived contrast and preference (a)(b) Results of Exp II using different dataset (c) Result of Exp I (d) Averaged result of Exp II

After the analysis of the relationship between the perceived contrast and the preference is completed, information on the lightness, chroma, and sharpness is used for contrast modeling: the standard deviation in the luminance channel for lightness, the standard deviation in the $\mathrm{C}^{*}{ }_{\mathrm{ab}}$ channel for chroma, and the standard deviation of image applied to the Sobel operator [7] in the luminance channel for sharpness. The results of the perceived contrast modeling are illustrated in Fig. 5.

As shown in Figs. 5 (b) and (c), the standard deviations of chroma and sharpness represented the relationship with the perceived contrast well. However, the standard deviation of lightness showed less correlation with the perceived contrast, and is a different result from that of [1-2]. The reason we had this result is that we used images of various lightness qualities, whereas the conventional experiments use only high-quality-lightness images. To determine the relationship between the contrast and 


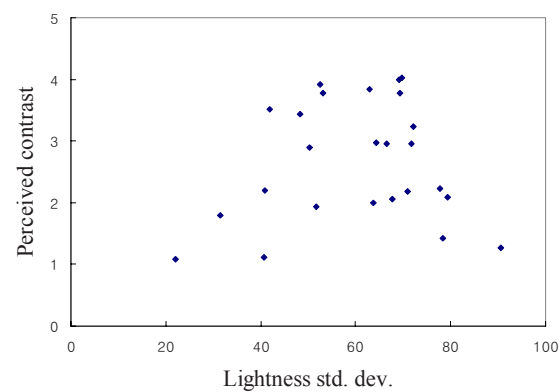

(a)

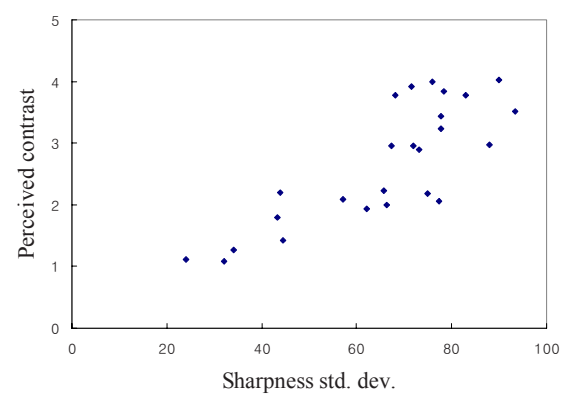

(c)

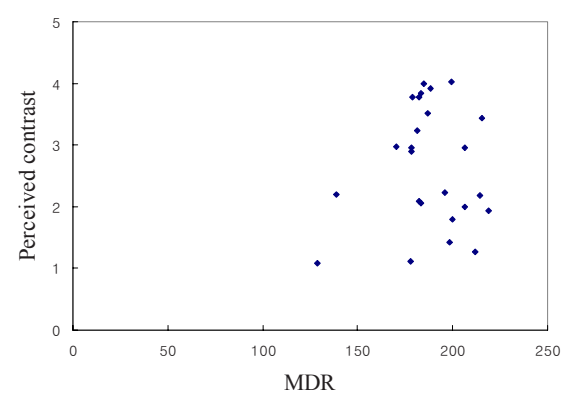

(e)

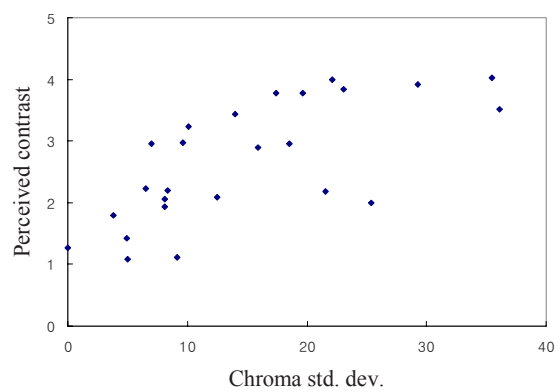

(b)

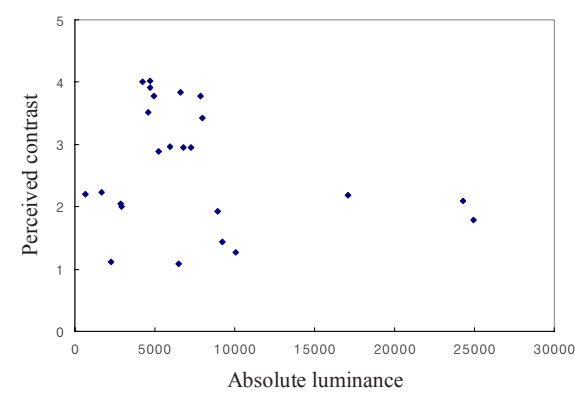

(d)

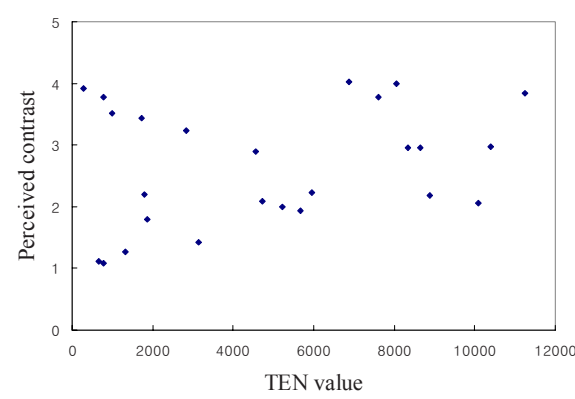

(f)

Fig. 5. The perceived contrast modeling. As the standard deviation of chroma (b) and sharpness (c) increase, the perceived contrast also increases. But it is difficult to find any relationship between contrast and the other values $(a)(d)(e)(f)$.

the luminance, we applied the absolute luminance [8] and the mean dynamic range (MDR) [9] as factors of lightness. Still, no good relationship could be derived. The TEN value [10] was also calculated as the sharpness measure, but the standard deviation of sharpness showed a better result. 
Finally, the standard deviations of chroma and sharpness were chosen and modeled owing to their high correlation with the perceived contrast. By using the least square estimation (LSE), the equations of estimated contrast for each experiment were defined as follows:

$$
\begin{aligned}
& \tilde{C}_{I}=0.0864 \times \sigma_{\text {chroma }}+0.0487 \times \sigma_{\text {sharpness }}-1.7983 \\
& \tilde{C}_{I I}=0.0365 \times \sigma_{\text {chroma }}+0.0329 \times \sigma_{\text {sharpness }}-0.205 .
\end{aligned}
$$

Eqs. (1) and (2) are the approximations of the results of Exp I and Exp II, respectively. By using these equations, we could model the contrast from the standard deviations of an image.

Next, we estimated the preference using the relationship with the perceived contrast. By using the least square regression method, the preference can be approximated by:

$$
\begin{gathered}
\tilde{P}_{I}=-0.3554 \times \tilde{C}_{I}^{2}+2.9004 \times \tilde{C}_{I}-1.7915 \\
\tilde{P}_{I I}=0.9679 \times \tilde{C}_{I I}+0.0844 .
\end{gathered}
$$

Eqs. (3) and (4) are calculated with a polynomial and a linear regression, respectively. Therefore, we could estimate the subjective image quality from an input image using these procedures.

To evaluate the validity of the experimental results, we procured test images and measured the image quality directly. The observers were asked to provide their subjective quality and contrast preferences in the form of discrete scores that were divided into five and marked according to the grade of an adjective: $\mathrm{Bad}=1$, Poor $=$ 2, Fair $=3$, Good $=4$, Excellent $=5$. It was not a pair comparison test, but rather, one image was displayed on a monitor for the observers. The condition of test is same as Exp I and II. A total of 24 observers participated in the test, and a total number of 85 test images were measured: 60 transformed images from five images similar to those

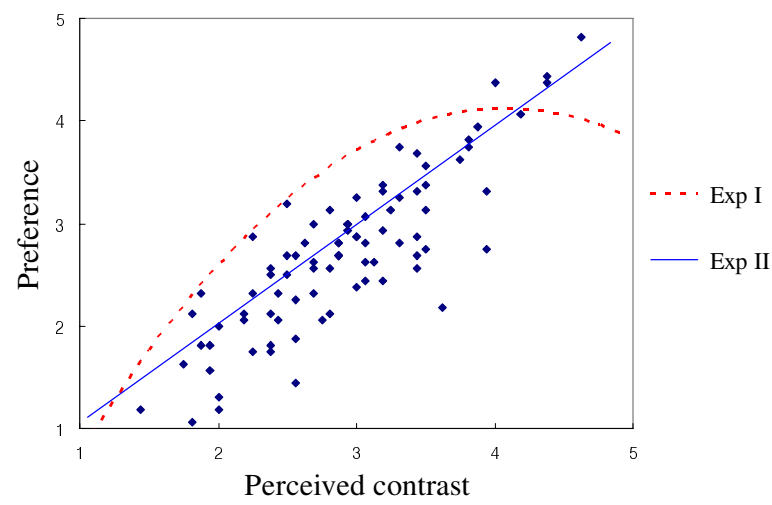

Fig. 6. The Relationship between the contrast and the preference with MOS 
used in Exp I, and 25 distinct images as Exp II. The resultant score being the criterion of quality verification, the mean opinion score (MOS) was calculated for each test image [4]. The MOS for contrast and the MOS for preference are plotted in Fig. 6.

The dashed and solid lines indicate the estimated results using Eqs. (3) and (4) respectively. We can easily see that solid line of Eq. (4) represents the MOS distribution with less error. This means that the contrast of image is the main factor determining subjective image quality.

For a more accurate comparison, we calculated the MSE with the estimated results for the image quality and the MOS. At the same time, the coefficient of determinant $\left(R^{2}\right)$ was computed for the suitability of the modeling. Each of the MSE and $R^{2}$ results are listed in Table 3.

Table 3. MSE and $R^{2}$ values for each experiment

\begin{tabular}{|c|c|c|c|}
\hline $\begin{array}{c}\text { Experiment } \\
\text { type }\end{array}$ & $\begin{array}{c}\text { MSE } \\
\text { (Contrast modeling) }\end{array}$ & $\begin{array}{c}\text { MSE } \\
\text { (Preference estimation) }\end{array}$ & $\boldsymbol{R}^{\mathbf{2}}$ \\
\hline Exp I & 0.8729 & 0.9685 & 0.7528 \\
\hline Exp II & 0.6615 & 0.5769 & 0.9475 \\
\hline
\end{tabular}

We could compare the performance of Exp II against Exp I within the $95 \%$ confidence intervals. The MSE of Exp II was reduced to 0.5769 , which is $40.4 \%$ lower than the result obtained by Exp I. We found that Exp. II with a higher $R^{2}$ offers a better modeling performance. These results showed, in other words, that the accuracy of Exp II is superior to that of Exp I. This means that an image with good contrast is considered to be a high-quality image, and that modeling using a dataset that consists of untransformed images yields a more reasonable result. Moreover, instead of an "inverted U" shape, the approximating preference in proportion to contrast is more reliable when we estimate the subjective image quality.

\section{Conclusions}

In this paper, we conducted two types of experiments for more accurate image-quality estimation. Whereas the conventional methods use only high-quality images for modeling, the dataset of Exp I included degraded images also. In Exp II, we compiled the dataset using different untransformed images, and modeled the subjective image quality. As a result of Exp I, the relationship between the perceived contrast and the preference manifested the "inverted U" shape, identically to the existing experiments. However, the Exp II result showed that the relationship between the perceived contrast and the preference was represented by a monotonically increasing function. Then, perceived contrast modeling was carried out using standard deviations of chroma and sharpness. Finally, we were able to estimate the subjective image quality, and the result showed that the approximated image quality showed an approximately 40\%-reduced MSE compared with the models that using transformed datasets. 
Further, our image-quality model can be applied in various fields in which digital image content is relevant, such as the digital camera and broadcasting system fields.

\section{References}

1. Calabria, A.J., Fairchild, M.D.: Perceived image contrast and observer preference I. The effects of lightness, chroma, and sharpness manipulations on contrast perception. Journal of Imaging Science and Technology 47, 479-493 (2003)

2. Calabria, A.J., Fairchild, M.D.: Perceived image contrast and observer preference II. Empirical modeling of perceived image contrast and observer preference data. Journal of Imaging Science and Technology 47, 494-508 (2003)

3. Kim, J.-S., Cho, M.-S., Koo, B.-K.: Experimental Approach for Human Perception Based Image Quality Assessment. In: Harper, R., Rauterberg, M., Combetto, M. (eds.) ICEC 2006. LNCS, vol. 4161, pp. 59-68. Springer, Heidelberg (2006)

4. Brotherton, M.D., Huynh-Thu, Q., Hands, D.S., Brunnstrom, K.: Subjective Multimedia Quality Assessment. IEICE Trans. Fundamentals E89-A, 2920-2931 (2006)

5. Ke, Y., Tang, X., Jing, F.: The Design of High-Level Features for Photo Quality Assessment. In: Proc. of IEEE Computer Vision and Pattern Recognition, pp. 419-426. IEEE Computer Society Press, Los Alamitos (2006)

6. Horita, Y., Sato, M., Kawayoke, Y., Parvez Sazzad, Z.M., Shibata, K.: Image Quality Evaluation Model Based on Local Features and Segmentation. In: Proc. of IEEE International Conference on Image Processing, pp. 405-408. IEEE Computer Society Press, Los Alamitos (2006)

7. Gonzalez, R.C., Woods, R.E.: Digital Image Processing, 2/E. Prentice-Hall, New Jersey (2002)

8. Akyuz, A.O., Reinhard, E.: Color appearance in high-dynamic-range imaging. Journal of Electronic Imaging 15(3), 033001 (2006)

9. Jourlin, M., Pinoli, J.C.: Image dynamic range enhancement and stabilization in the context of the logarithmic image processing model. Signal Processing 41(2), 225-237 (1995)

10. Buerkle, A., Schmoeckel, F., Kiefer, M., Amavasai, B.P., Caparreli, F., Selvan, A., Travis, J.R.: Vision-based closed-loop control of mobile microbots for microhandling tasks. Proc. SPIE 4568, 187-198 (2001) 